\title{
Chinese herbal formulas for treating hypertension in traditional Chinese medicine: perspective of modern science
}

\begin{abstract}
Xingjiang Xiong ${ }^{1,3}$, Xiaochen Yang ${ }^{1}$, Yongmei Liu ${ }^{1}$, Yun Zhang ${ }^{1}$, Pengqian Wang ${ }^{2,3}$ and Jie Wang ${ }^{1}$
Hypertension, which directly threatens quality of life, is a major contributor to cardiovascular and cerebrovascular events. Over the past two decades, domestic and foreign scholars have agreed upon various standards in the treatment of hypertension, and considerable progress has been made in the field of antihypertensive drugs. Oral antihypertensive drugs represent a milestone in hypertension therapy. However, the blood pressure standard for patients with hypertension is far from satisfactory. The study of Chinese herbal formulas for treating hypertension has received much research attention. These studies seek to integrate traditional and Western medicine in China. Currently, Chinese herbal formulas are known to have an outstanding advantage with regard to bodily regulation. Research shows that Chinese medicine has many protective mechanisms. This paper addresses the process of the antihypertensive mechanisms in Chinese herbal formulas for treating hypertension. These mechanisms are to be discussed in future research.
\end{abstract}

Hypertension Research (2013) 36, 570-579; doi:10.1038/hr.2013.18; published online 4 April 2013

Keywords: Chinese herbal formulas; traditional Chinese medicine; treatment efficacy

\section{INTRODUCTION}

Cardiovascular and cerebrovascular events are the major causes of mortality worldwide. ${ }^{1}$ Hypertension, a chronic disease in which the blood pressure (BP) in the arteries is elevated, is a major contributor to vascular morbidity and mortality. Patients with hypertension are more likely to have heart, brain, kidney and peripheral vascular diseases than those with normal BP. Over the past two decades, domestic and foreign scholars have agreed upon various standards in the treatment of hypertension. Robust evidence from randomized trials shows that the treatment of hypertension is remarkably effective, and a small reduction in BP might cause a large reduction in the risk of cerebrovascular event and myocardial infarction. ${ }^{2}$ Oral antihypertensive drugs represent a milestone in therapy for hypertension and other cardiovascular diseases (CVDs); furthermore, they provide the primary and secondary prevention strategies to combat these diseases. ${ }^{3}$ In addition, maintaining an active lifestyle, improving diet, monitoring total caloric intake and practicing adequate exercise have pivotal roles in hypertension treatment. Therapeutic strategies are based on the following principles. (a) Early intervention includes the prevention and treatment of pre-hypertension. In addition, the goal of therapy has changed from 'the lower, the better' to 'the earlier, the better', which coincides with ancient preventive medicine (that is, 'treat what is not yet ill'; the 'Huangdineijing' and 'Nanjing' classics in traditional Chinese medicine (TCM) theory). ${ }^{4}$ This proverb promotes interventions to the internal organs that are not affected by the ongoing morbidity process and can be compared with avoiding target-organ damage (TOD) due to sustained high BP. (b) In a comprehensive intervention, the treatment focuses on risk factors and includes optimal antihypertensive strategies, such as antihypertensive therapy combined with lipid-lowering therapy or antihypertensive therapy combined with homocysteine-lowering therapy. (c) The therapeutic target changes from the simple goal of lowering BP to improving the complications associated with TOD to reduce the long-term risk of CVD, thereby achieving an ideal BP. (d) Furthermore, the treatment emphasizes on effectively controlling $\mathrm{BP}$, especially with regard to reducing $\mathrm{BP}$ variability $(\mathrm{BPV}) .^{5}$

Considerable progress has been made in the field of antihypertensive drugs. For example, renin-angiotensin-aldosterone system (RAAS) has a crucial role in the regulation of BP and cardiovascular remodeling. The RAAS inhibitor is currently considered an important cornerstone of reducing the risk of cardiovascular events. ${ }^{6}$ Aside from the commonly used oral antihypertensive drugs, such as angiotensinconverting enzyme inhibitor (ACEI), angiotensin II type 1 receptor

${ }^{1}$ Department of Cardiology, Guang'anmen Hospital, China Academy of Chinese Medical Sciences, Beijing, China and ${ }^{2}$ Department of Endocrinology, Traditional Chinese Medicine Hospital of Mentougou District, Beijing, China

${ }^{3}$ These authors contributed equally to this paper.

Correspondence: Dr X Xiong or X Yang or Dr J Wang, Department of Cardiology, Guang'anmen Hospital, China Academy of Chinese Medical Sciences, Beixiange 5\#, Xicheng District, Beijing 100053, China.

E-mail: 5administration@163.com or avill1988@126.com or wangjie0103@yahoo.cn

Received 4 July 2012; revised 26 November 2012; accepted 12 December 2012; published online 4 April 2013 
(AT1R) blockers, beta blockers and aldosterone receptor antagonist, new RAAS inhibitors have been recently researched and developed. For instance, Aliskiren, the first orally active direct rennin inhibitor, is a novel antihypertensive drug that inhibits the first step of RAAS. It blocks the conversion of angiotensinogen to angiotensin I (Ang I) to reduce the secretion of Ang II. ${ }^{7}$ Compared with ACEI and ARB, direct rennin inhibitors stop the 'ACE escape phenomenon' and do not influence the plasma rennin activity during long-term use. ${ }^{8-10}$ Furthermore, angiotensin II type 2 receptor, a new type of RAAS inhibitor, can prevent the production of anti-vascular smooth muscle cells (anti-VSMC), relax blood vessels and reverse cardiovascular remodeling. Aldosterone synthase (CYP11B2) inhibitors can inhibit the final step of biochemical reactions catalyzed by the enzymes of in vivo aldosterone synthesis process, thereby reducing aldosterone synthesis. Preventing the reactive elevation of aldosterone, compared with aldosterone receptor antagonist, is a new way to antagonize aldosterone. In addition, a significant amount of valuable research has come from modern medicine and pharmacology. Recently, other, newer agents, including a antihypertensive vaccine against Ang I, Ang II, AT1R, endothelin receptor antagonist (Bosantan, Darusentan, Siaxsentan and Tezosentan), chymase, Ang 1-7, neutral endopeptidase-ACE dual inhibitors (omapatrilat, sampatfilt) and so on, are being developed. Despite their proven benefits, achieving an ideal BP level in patients with hypertension is not satisfactory because only $25 \%$ of patients achieve this goal, and recurrent cardiovascular events still occur in those who take antihypertensive drugs. At the same time, numerous adverse reactions, including headache, dizziness, orthostatic hypotension and decreased sexual function, limit the clinical practice of antihypertensive drugs. Thus, reducing the occurrence and development of hypertension is necessary to prevent TOD and decrease the vascular morbidity and mortality related to hypertension. ${ }^{11,12}$ Developed novel classes of antihypertensive agents must possess high efficacy. As always, fewer adverse effects are the research focus to prevent CVD.

The study of Chinese herbal formulas for treating hypertension is the most active area of research within TCM and integrative medicine in China. Over the past 30 years, significant progress has been made from theory and experiments in the clinical fields based on the inheritance and innovation of thoughts in TCM, to clarify the treatment regulations and principles of hypertension. Currently, much attention has been paid to the holistic, multitarget and multidimensional pharmacological studies of TCM. ${ }^{13,14}$ Moreover, the efficacy of TCM for treating hypertension is demonstrated by numerous published cases and randomized trials. ${ }^{15-17}$ However, some trials have found null results. ${ }^{18-21}$ Numerous randomized controlled trials have been conducted in China to evaluate the efficacy of novel Chinese herbal formulas generated from well-known Chinese medicine formulas or from currently effective practices to treat hypertension. Some trials have effectively treated hypertension. ${ }^{22,23}$ An increasing number of systematic reviews and meta-analyses have been conducted to assess the efficiency of TCM for treating hypertension, providing the best evidence supporting the use of TCM for patients who suffer from hypertension. ${ }^{24-34}$ The majority of these studies have positive findings that favor the practice of TCM. These studies reveal the multi-protective mechanisms of Chinese herbal formulas for treating hypertension. A recent study showed a strong association between TCM syndrome (also called 'zheng' or 'pattern') and hypertension. ${ }^{35}$ Thus, Chinese herbal formulas, including classical prescriptions, experienced prescriptions, traditional Chinese patent medicine and others based on syndrome differentiation, were analyzed further with regard to the aspects shown in Table 1.

\section{THE ANTIHYPERTENSIVE MECHANISM OF CHINESE HERBAL FORMULAS FOR TREATING HYPERTENSION}

\section{Reduce BPV}

For many decades, the major goal of antihypertensive treatment was to lower BP to a defined level. Recently, several investigators have shown that BPV is another critical cardiovascular risk factor that should also be emphasized in the treatment of hypertension. ${ }^{36} \mathrm{BPV}$ refers to the physiological fluctuations of BP over time that result from the complex interactions of the dynamic regulations of in vivo neuroendocrine systems. These interactions are multifaceted phenomena that include both short- and long-term components that can be estimated using the s.d. of the BP values over a day or using the night-to-day BP ratio. Studies show that, aside from increasing average $\mathrm{BP}$, the load and circadian rhythm of $\mathrm{BP}$ are closely associated with cardiac and vascular remodeling as well as TOD. ${ }^{37}$ If patients clearly have TOD, then their BPV will increase because of their constantly high systolic pressure at night. Furthermore, increased BPV is associated with a higher incidence of the cardiovascular morbidity complications related to hypertension. The mechanisms of BPV that lead to TOD might be relevant to vascular endothelial injury, RAAS activation, inflammatory response stimulation and the acceleration of target organ apoptosis. Because of the relevant predictions of these associated mechanisms, the adverse prognostic significance of increased BPV has clinical experts increasingly concerned.

Recent studies have shown that various syndromes are highly correlated with BPV. Liver fire (Gan huo shang yan)/liver-yang hyperactivity (Gan yang shang kang) syndrome and phlegm-fluid retention (tan zhuo nei $z u$ ) syndrome are two major types of hypertension discussed in TCM. Compared with patients who suffer from liver fire/liver-yang hyperactivity syndrome, patients with phlegm-fluid retention syndrome have more significant BP day-night circadian reductions and BP overload increases. Furthermore, it might injure target organs easily. ${ }^{38}$ Chinese herbal formulas for treating liver fire/liver-yang hyperactivity syndrome and phlegm-fluid retention syndromes, such as Qingxuanjiangya decoction, ${ }^{39,40}$ Niuhuangjiangya pill, ${ }^{41}$ Songlingxuemaikang capsule ${ }^{42-44}$ and Banxia baizhu tianma decoction, ${ }^{45}$ can reduce $\mathrm{BPV}$, control $\mathrm{BP}$ and improve clinical symptoms both in vitro and in vivo.

\section{Inhibiting the activity of sympathetic nerve}

Does sympathetic activation initiate and maintain BP elevation in patients with hypertension? Strong historical and contemporary evidence supports this claim. The chronic activation of the sympathetic nervous system is the dominant contributor to systemic hypertension that directly leads to elevation and fluctuation of BP by increasing renin release and cardiac output as well as by promoting vasoconstriction. Therefore, maintaining BP by suppressing sympathetic activity and balancing the sympathetic vagus nerve system is necessary. ${ }^{46,47}$

Verticil, also known as the alkaloid of Rauwolfia verticallata, is an antihypertensive drug that is independently produced in China. It consists of reserpine and ingredients such as $\alpha$-receptor blockade. In addition, both berberine and isoliensinine have a strong effect with regard to blocking the $\alpha 1$ receptor that can reduce BP and the left ventricular mass/body mass ratio in renovascular hypertensive rats (as shown in Figures 1 and 2).48,49 Other TCM chemical components, rhynchophylline and isorhynchophylline, can dilate peripheral vessels by 


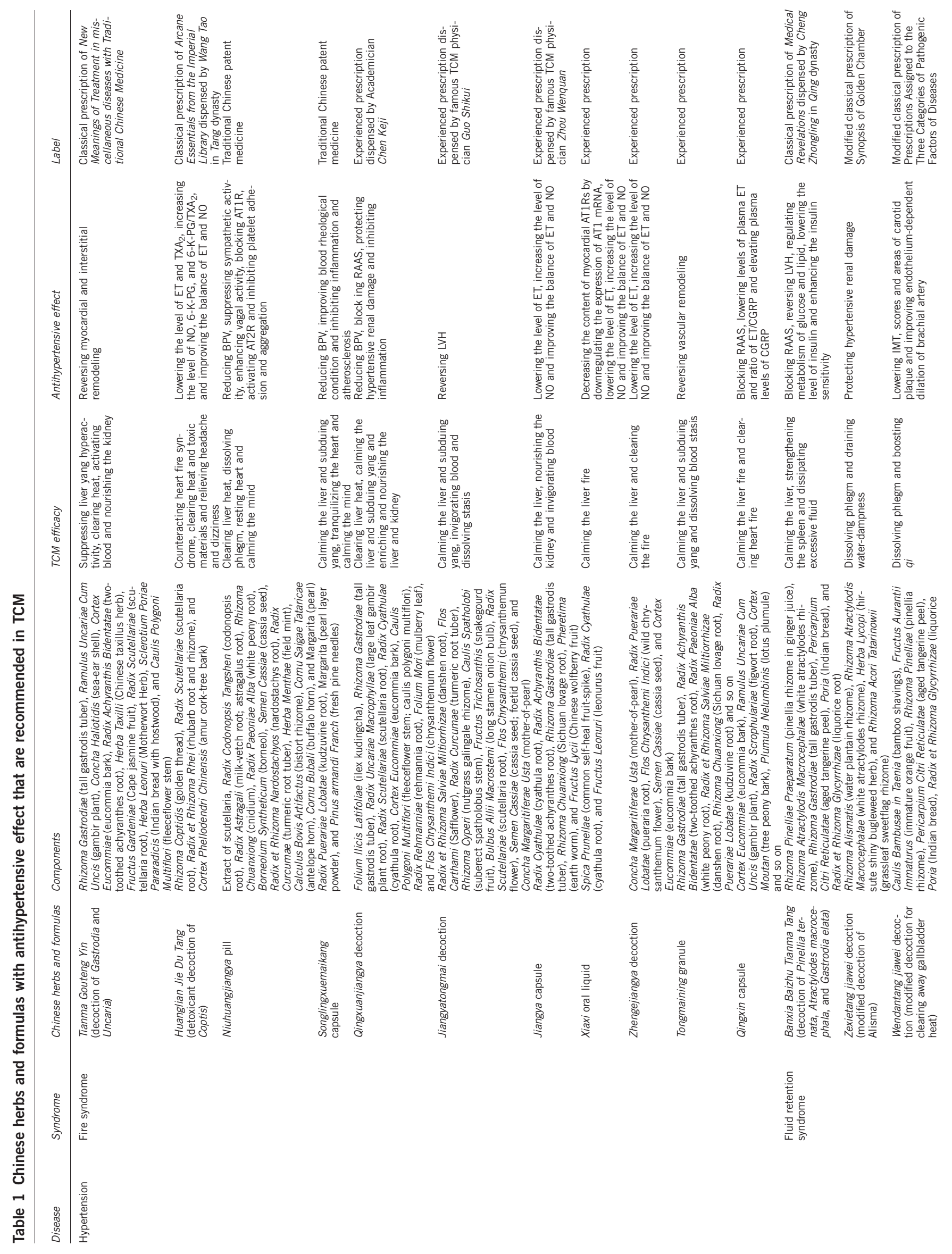




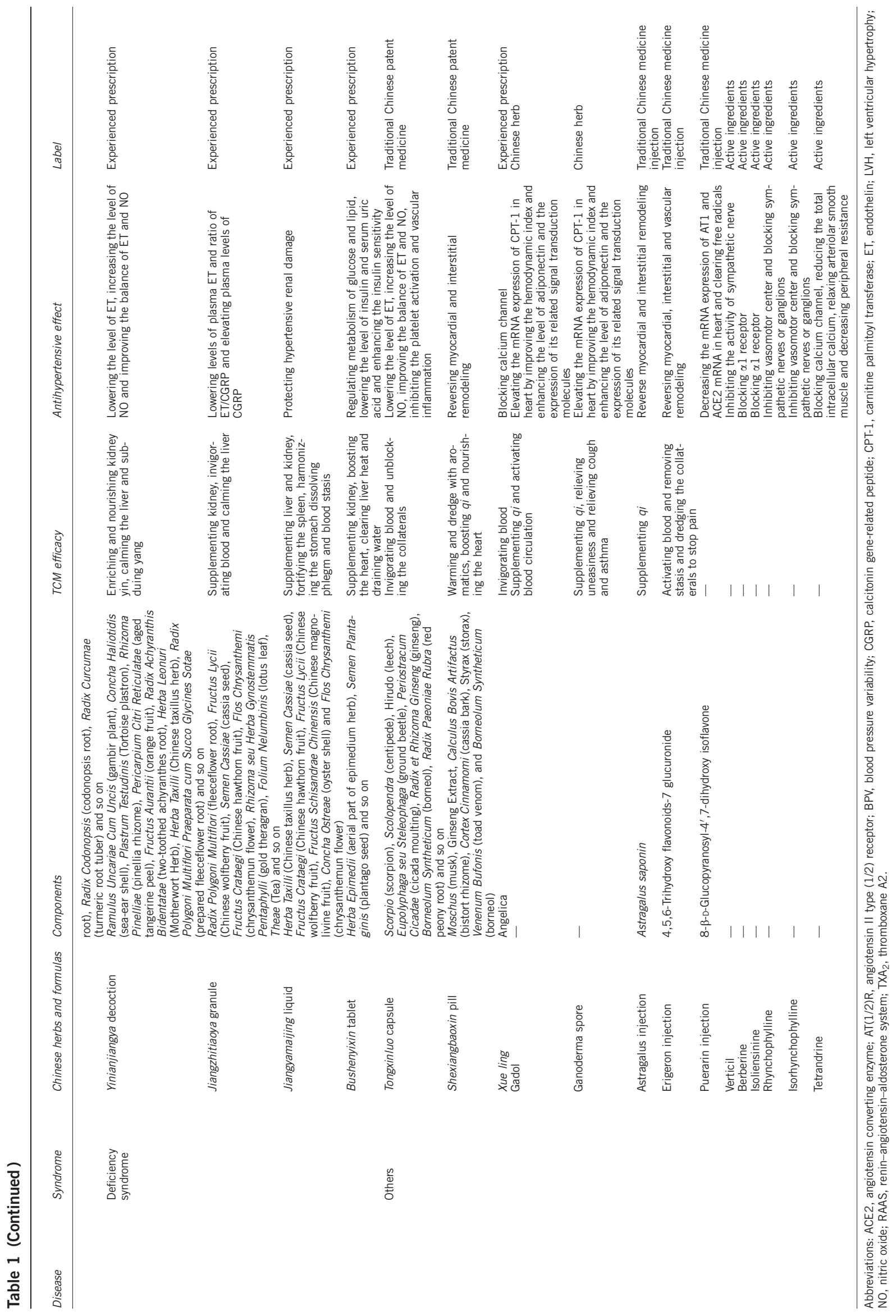


<smiles>COc1ccc2cc3[n+](cc2c1OC)CCc1cc2c(cc1-3)OCO2</smiles>

Figure 1 Chemical structure of berberine.<smiles>COc1ccc(C[C@H]2c3cc(Oc4cc(C[C@@H]5c6cc(O)c(OC)cc6CCN5C)ccc4O)c(OC)cc3CCN2C)cc1</smiles>

Figure 2 Chemical structure of isoliensinine.<smiles>CCC1CN2CCC3(C(=O)Nc4ccccc43)C2CC1/C(=C\OC)C(=O)OC</smiles>

Figure 3 Chemical structure of rhynchophylline.<smiles>CCC1=C(/C(=C\OC)C(=O)O)CC2CN3CCC3(C(=O)Nc3ccccc3)C2C1</smiles>

Figure 4 Chemical structure of isorhynchophylline.

directly inhibiting the vasomotor center and blocking the sympathetic nerves or ganglions (as shown in Figures 3 and 4). ${ }^{50}$ Niuhuangiiangya pill can suppress sympathetic activity and enhance vagal activity, thereby significantly lowering plasma rennin activity and epinephrine after a grip-strength test to stabilize BP under stress and effectively control 24-h BP levels. ${ }^{51}$

\section{Blocking the renin-angiotensin system}

For the past three decades, the renin-angiotensin system has been a major focus in high BP research. RAAS has an important role in the acute and chronic regulation of BP. Excessive RAAS activation not only causes sustained increases in BP but also leads to arterial vasoconstriction, fibrosis and cardiac remodeling. Ang II, a crucial vasoactive peptide, which is one of the strongest hormones among the endogenesis active peptides used to reduce BP, is involved in

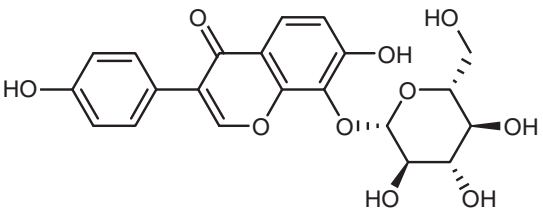

Figure 5 Chemical structure of Puerarin.

vasoconstriction as well as the proliferation and migration of smooth muscle cells, formation of foam cells, aggregation and adhesion of platelets, bradykinin degradation, nitric oxide (NO) reduction and endothelin (ET) increases by acting on AT1R. ${ }^{52}$ Qingxuanjiangya decoction and Qingxin capsule might safely and effectively lower BP in patients with mild or moderate degrees of hypertension, improve their clinical symptoms and more effectively improve their quality of life. This mechanism might be related to their ability to inhibit the activity of the circulatory renin-angiotensin system. ${ }^{39,53}$

AT1R activation can cause arterial vasoconstriction, smooth muscle cell proliferation and strengthen myocardial contraction, which can lead to hypertension, vascular remodeling and cardiac hypertrophy. ${ }^{54}$ Several studies have demonstrated that Xiaxi oral liquid lowers BP and the content of myocardial AT1Rs by downregulating the expression of AT1 mRNA in spontaneously hypertensive rats (SHR). ${ }^{55}$ Furthermore, the antihypertensive effect of Niuhuangjiangya pill, another traditional Chinese patent medicine, is related to directly blocking AT1R and indirectly activating angiotensin II type 2 receptor. ${ }^{56,57}$

ACE2 is a zinc-dependent metalloproteinase that has a strong homology with ACE. In addition, ACE2 is similar to the endogenous ACEI that can degrade ACE substrates and decompose ACE products such as Ang II (1-7), thereby resulting in lowered BP and inhibited myocardial hypertrophy. Much research has shown that Banxia Baizhu Tianma Tang (a decoction of Pinellia ternata, Atractylodes macrocephala and Gastrodia elata) can improve the expression of factors in cardiac RAAS via a dynamic long-term process. The expression of ACE2 mRNA gradually increases with the extension of delivery time. Importantly, the effect was similar with captopril..$^{58}$ Puerarin, also called $4^{\prime}-7$-dihydroxy-8-beta-D-glucose isoflavones, is the major active ingredient extracted from the roots of the kudzuvine, which induces anti-platelet aggregation, activates blood vessels and lowers BP (as shown in Figure 5). Study has demonstrated that low dose of puerarin decreases the mRNA expression of AT1 and ACE2 mRNA in the heart, whereas high doses increase these expression levels in the kidney. A feedback correlation might exist between AT1 and ACE2. ${ }^{59}$

\section{Improving endothelial function}

Vascular endothelial factors also have a crucial role in the regulation of BP. Under the stimulation of hypertension, vessel endothelial cells (VEC) release a series of endothelial growth factors that can result in the proliferation and hypertrophy of VSMC, increase intimal collagen, thicken vascular walls and increase peripheral vascular resistance. Finally, all these actions contribute to a vicious circle. ${ }^{60,61}$ The research concerning VEC, an initiating factor and carrier of the 'endothelium-hypertension-cardiovascular event' chain, has become a hot issue in the field of hypertension. Thus, early endothelial dysfunction interventions can delay and control the development of cardiovascular and cerebrovascular events.

VEC-stimulated synthesis releases a variety of vasoactive substances through paracrine, autocrine and endocrine systems to regulate 
vascular tone, stimulate smooth muscle cell growth and proliferation, promote blood/endothelial cell adhesion, reverse vascular remodeling and participate in blood coagulation, fibrinolysis and immune system. ${ }^{62}$ The endothelium-dependent contraction factors produced and released by VEC include ET-1, thromboxane A2 (TXA 2 ), Ang II, asymmetric dimethyl arginine, $\mathrm{O}^{2-}$ (superoxide anion $\left(\mathrm{O}^{2-}\right)$ ), urotensin and coupling factor 6. The endothelium-dependent relaxing factors produced and released by the VEC include NO, prostacyclin $\left(\mathrm{PGI}_{2}\right)$, calcitonin gene-related peptide (CGRP), endothelium-derived hyperpolarizing factor and C-type natriuretic peptide.

NO, which is synthesized by endothelial nitric oxide synthase, maintains vasodilatation and contraction as well as a balance between anticoagulants and procoagulants. ${ }^{63} \mathrm{ET}$ is a strong vasoconstrictor that can produce long-lasting concentration-dependent vasoconstriction and cause vascular spasms. ${ }^{64}$ It can also stimulate VSMC proliferation, thicken the vascular wall in atherosclerosis and eventually lead to high BP and vascular structural changes. Therefore, the imbalance between the synthesis and release of NO and ET-1 is one of the most important mechanisms in the occurrence and development of hypertension. In addition, this balance is a characteristic of VEC damage. Several studies have demonstrated that certain classic prescriptions lower the level of ET while increasing the level of $\mathrm{NO}$ and improve the balance of ET and NO, prevent endothelial injury and improve the peroxidation pathological hyperactivity state in the cardiovascular system of patients with hypertension. These types of classic prescriptions include Huanglian Jie Du Tang (Arcane Essentials from the Imperial Library written by Wang Tao in Tang dynasty), ${ }^{65}$ Xiaxi oral liquid, ${ }^{66}$ Tongxinluo capsule, ${ }^{67}$ Zhengejiangya decoction, ${ }^{68}$ Yinianjiangya decoction ${ }^{69,70}$ and Jiangya capsule. $^{71-73}$

CGRP is the strongest vasodilator, and it has a crucial role in the systolic and diastolic functions of the cardiovascular system. CGRP fights against the vascular effects of ET and Ang II, enhances the left ventricular systolic function and reduces myocardial ischemia. Both Qingxin capsule and Jiangzhitiaoya granule ${ }^{74}$ could lower levels of plasma ET and the ET/CGRP ratio and elevate the plasma levels of CGRP.

$\mathrm{PGI}_{2}$ is the strongest in vivo platelet depolymerization and vasodilator. 6-keto-prostaglandin $\mathrm{F}_{1 \alpha}\left(6-\mathrm{K}_{-} \mathrm{PGF}_{1 \alpha}\right)$ is a metabolite of $\mathrm{PGI}_{2}$ that may indicate the content of $\mathrm{PGI}_{2}$ because of its stable nature. $\mathrm{TXA}_{2}$ is a strong platelet aggregation substance and vasoconstrictor. Therefore, $\mathrm{PGI}_{2}$ and $\mathrm{TXA}_{2}$ are important vasoactive substances in the regulation of vascular wall tension. The balance between these elements has an important role in maintaining hemodynamics and vasoconstriction. Studies have shown that Huanglian Jie Du Tang can increase the levels of 6-keto-prostaglandin (6-K-PG) and 6-K-PG/ $\mathrm{TXA}_{2}$ while lowering TXA 2 in SHR. These findings indicate that $\mathrm{PGI}_{2}$ and $\mathrm{TXA}_{2}$ improve hemodynamics and maintain the balance between the coagulation and anticoagulation. ${ }^{65}$

\section{Preventing TOD}

Given the progress of research on hypertension, the structure and function of target organs such as the heart, brain, kidneys and blood vessels have been reconsidered to certain extent. Consequently, much attention should be paid to preventing and treating TOD while lowering BP. ${ }^{75}$ Recent research showed that a strong association exists between TCM syndromes and the clinical symptoms of TOD. ${ }^{35}$ That is, a liver-fire blazing upward pattern was related to cerebrovascular and eye disease and heart disease (HD); the obstruction of phlegm and the dampness of the heart/liver/gallbladder pattern were related to kidney disease; $q i$ and blood deficiencies leading to liver-yang rising patterns were related to HD and kidney disease; and kidney yin/yang deficiency patterns were related to cerebrovascular and eye disease and HD.

Left ventricular hypertrophy is often assumed to be a serious TOD for hypertension. In fact, left ventricular hypertrophy is an independent risk factor for sudden death, coronary HD, congestive heart failure, arrhythmia and other cardiovascular events. Because of the increased long-term pressure load, catecholamine, Ang II and other growth factors can stimulate myocardial hypertrophy and interstitial fibrosis. Therefore, simply lowering BP might not solve the problem of TOD. Furthermore, reversing left ventricular hypertrophy, improving cardiac function and protecting target organs have become the primary goals of hypertension treatment. ${ }^{76-78}$ Gadol (Sedum rhodiola medicinal plants) and Ganoderma spore (the dissemination of spores during the maturation of the medicinal fungus Ganoderma lucidum fruiting bodies) might treat a variety of CVDs. Gadol and Ganoderma spore medications, either alone or in combination, might significantly reduce systolic BP, diastolic BP and the myocardial hypertrophy index as well as elevate the mRNA expression of carnitine palmitoyl transferase in the heart by improving the hemodynamic index in SHR, thereby enhancing the level of adiponectin and the expression of its related signal transduction molecules. ${ }^{79}$ In addition, liver fire/liveryang hyperactivity syndrome is strongly related to HD in TOD. Chinese herbal medicine such as Banxia Baizhu Tianma Tang, Jiangyatongmai decoction and Tianma Gouteng Yin, which are used to treat liver fire/liver-yang hyperactivity syndrome, all have potential effects on HD. Banxia Baizhu Tianma Tang might significantly decrease the cardiac hypertrophy of 18-week and 24-week-old SHR by regulating the mRNA expression of the RAS factor. The therapeutic effect of this herbal medicine is similar to that of captopril. $^{58}$ Jiangyatongmai decoction, a Chinese medicine for invigorating blood circulation and eliminating blood stasis, is a historical prescription dispensed by the famous TCM doctor Guo Shikui. This herb might reduce BP, reverse left ventricular remodeling and enhance left ventricular function by inhibiting the AKT protein, decreasing the levels of ET and Ang II and increasing the level of CGRP. ${ }^{80,81}$ In addition to substantial myocardium reconstruction, myocardial collagen remodeling is common in hypertension. Much research has demonstrated that Tianma Gouteng Yin (a decoction of Gastrodia and Uncaria), ${ }^{82-84}$ Astragalus injection, ${ }^{85}$ Erigeron injection ${ }^{86}$ and Shexiangbaoxin pill $^{87}$ can reverse myocardial and interstitial remodeling and significantly reduce perivascular collagen area, the levels of left ventricular mass index, left ventricular wall thickness and interventricular septum thickness, the content of Type I and Type III collagen, the diameter of myocardial cells, myocardial collagen synthesis and extracellular matrix deposition. This mechanism might be related to the reduction in myocardial transforming growth factor- $\beta 1$ expression.

Hypertensive renal injury is another major TOD. Long-term hypertension can cause renal sclerosis and gradually progress to chronic renal failure. Positive control of hypertension is the key to preventing hypertensive renal damage. According to recent studies, hypertensive renal injury is strongly related to fluid, phlegm and dampness retention syndrome and liver-yang hyperactivity syndrome, which are caused by deficiency syndrome. Chinese medicines such as Zexietang jiawei decoction (a modified decoction of Alisma), Jiangyamaijing liquid and Qingxuanjiangya decoction, which are used to treat fluid, phlegm and dampness retention syndrome, deficiency syndrome and liver-yang hyperactivity syndrome, respectively, have certain advantages with regard to treating hypertensive renal injury. 
Clinical research indicates that prescriptions such as Zexietang jiawei decoction, ${ }^{88}$ Jiangyamaijing liquid ${ }^{89}$ and Qingxuanjiangya decoction ${ }^{90}$ might control increased systolic BP, inhibit the glomerular and tubular hyperplasia caused by high BP in SHR and significantly reduce urinary albumin and $\beta 2$-microglobulin by increasing the activity of renal rennin and the level of Ang II.

Vascular remodeling is a series of adaptive structural and functional changes in the blood vessels caused by hemodynamics and humoral factors. In addition, it primarily manifests as VSMC hypertrophy and hyperplasia, increased perivascular collagen, decreased vascular compliance and changes in its reactivity to vasoactive substances. Furthermore, the structure and functional changes of large vessels might lead to atherosclerosis. Recent studies have shown that the active ingredients of Chinese medicine might improve vascular remodeling by regulating RAAS and inhibiting a variety of cytokines (inflammatory factors). ${ }^{91,92}$ Erigeron injection ${ }^{86}$ and Tongmaining granule $^{93}$ might reduce perivascular Type I collagen, improve vascular compliance and reverse vascular remodeling through the inhibition of PKC activity in addition to lowering BP. Other studies have found that Wendantang jiawei decoction (a modified decoction for clearing away gallbladder heat and a classic TCM prescription for treating phlegm turbid retention syndrome) might significantly lower intima-media thickness scores and carotid plaque areas as well as improve the endothelium-dependent dilation of the brachial artery. ${ }^{94}$

\section{Improving insulin resistance as well as glucose and lipid metabolism}

Hypertension is often associated with lipid and glucose metabolism disorders. The interaction between these disorders might continue to diminish arterial elasticity, increase peripheral resistance and change hemorheology, thereby leading to microcirculation disturbance and high rates of CVD. Therefore, intervening and treating risk factors such as impaired glucose tolerance, dyslipidemia, abdominal obesity and hyperhomocysteinemia are equally important in hypertension treatment. ${ }^{95,96}$ In addition, several recent studies have shown that the fasting insulin levels in patients with hypertension were significantly higher than normal. Moreover, insulin resistance is one of the most important aspects of the pathological basis of hypertension. Clinical studies have also shown that Chinese herbal formulas for treating hypertension, such as Puerarin, Banxia baizhu tianma Tang and Bushenyixin pill, might reverse the risk factors of hypertension, regulate glucose and lipid metabolisms, lower insulin levels and enhance insulin sensitivity. ${ }^{97-101}$ Furthermore, Puerarin has a crucial role in eliminating free radicals. ${ }^{98}$ Banxia baizhu tianma Tang might improve salt sensitivity and lower cholesterol, low-density lipoprotein cholesterol, triglycerides and the body mass index of patients with hypertension and abundant phlegm-dampness syndrome..$^{99,100}$ Bushenyixin pill might reduce the serum uric acid level in patients with essential hypertension and insulin resistance. ${ }^{101}$

\section{Other mechanisms}

In addition to the above mechanisms, calcium ions have a vital role in the development of hypertension. The activation of potentialdependent calcium channel and receptor regulation of calcium channel might cause an extracellular calcium influx, release of intracellular calcium and lead to a higher concentration of intracellular free $\mathrm{Ca}^{2+}$. Chinese herbal formulas also have calcium channel antagonists and lower BP. Tetrandrine, a well-known extract of Fangji (Radix stephaniae tetrandrae), is both a natural, non-selective calcium channel blocker and a calmodulin antagonist; moreover, it lowers BP by blocking calcium channels, thereby reducing total intracellular calcium, relaxing arteriolar smooth muscle and decreasing peripheral resistance. ${ }^{102}$ In addition, Xue ling, the primary component of angelica, has a similar effect on verapamil, which indicates that antihypertensive activity is also associated with calcium channel blocking. ${ }^{103}$

Chinese herbal formulas might also improve blood rheological conditions, such as blood flow, viscosity, deformability and coagulation, in patients with hypertension. Because of the enhancement of platelet adhesion, aggregation, releasing reaction and the erythrocyte deformability dysfunction in patients with hypertension, red blood cell deformability improvement and platelet activation inhibition might help in treating patients with hypertension. Studies show that Songlingxuemaikang capsule improves the hemorheology of patients with hypertension, especially high shear blood viscosity, plasma viscosity and whole-blood-reduced viscosity. ${ }^{104}$ Tongxinluo capsule inhibits the platelet activation and vascular inflammation of essential hypertension patients with comorbid diabetes and reduces the levels of serum high-sensitivity C-reactive protein (Hs-CRP), plasma fibrinogen C (FIB-C), CD62p and glycoprotein b/a (GP b/a). ${ }^{67}$ Niuhuangjiangya pill significantly inhibits platelet adhesion and aggregation in normal rats by inhibiting the release of the $\mathrm{TXA}_{2}$ platelets induced by adenosine diphosphate, which might block platelet activation and aggregation as well as regulate the positive feedback between the release of adenosine diphosphate and $\mathrm{TXA}_{2}{ }^{105}$

The important role of inflammation in the incidence and complications of CVD has received much attention. Inflammation, which most likely acts as a trigger and may be associated with CRP to a large extent, tumor necrosis factor- $\alpha$ and other related inflammatory factors most likely have vital roles in the process of remodeling of the vessels, myocardium and renal interstitial cells in patients with hypertension. For instance, Qingxuanjiangya decoction not only lowers Hs-CRP and inhibits the inflammatory response but also regulates lipid metabolism and increases the level of endogenous estrogen in perimenopausal women. Furthermore, Qingxuanjiangya decoction can reduce the occurrence of cardiovascular events in addition to lowering BP. Songlingxuemaikang capsule has a better therapeutic effect than Qingxuanjiangya decoction for inflammatory and carotid atherosclerosis, which reduces the content of Hs-CRP and the atherosclerotic plaque index in patients with hypertension. ${ }^{106}$ Niuhuangjiangya pill also has a role in anti-atherosclerosis. The tumor necrosis factor among patients with atherosclerosis was significantly lower after taking this medicine. ${ }^{107}$

\section{DISCUSSION AND PERSPECTIVES}

TCM has a long history and abundant experience with regard to treating the clinical manifestations frequently reported by patients with hypertension and probable TOD. ${ }^{108,109}$ With the increasing popularity of complementary and alternative medicines among patients with hypertension, ${ }^{110-112}$ TCM is being more frequently used in China and the West. ${ }^{113,114}$ TCM also has a unique way of diagnosing and treating this disease. Furthermore, a variety of TCM practices, including Chinese herbal formulas, acupuncture, moxibustion, cupping, qigong, Tai Chi (a shadow-boxing exercise), diet and exercise therapy, originated in China. ${ }^{115-121}$ Among these practices, Chinese herbal formulas are a key research area. Great amount of effective Chinese herb and formulas including classical prescriptions originated from a 'classic' textbook, experienced prescriptions dispensed by famous TCM physicians, traditional Chinese patent medicines and others have been widely used in clinical practice by TCM practitioners for thousands of years. Under the guidance of holistic concept and treatment based on 
syndrome differentiation and formula syndrome differentiation, ${ }^{122}$ TCM practitioners diagnose certain syndromes and prescribe certain formulas according to the clinical manifestations of patients with hypertension. Thus, corresponding Chinese herbal formulas are prescribed based on a TCM diagnosis. In most cases, selected classical prescriptions should be modified (either added or deleted) based on individual symptoms. Current research demonstrates that Chinese herbal formulas possess the advantage of whole body regulation in many ways for many targets. Recently, the continued study of the anti-hypertensive mechanisms of Chinese herbal formulas for hypertension has made great progress with regard to the etiology and pathogenesis of this disease. In addition, progress has been made with regard to treatment regulations and the principles of antihypertensive drugs as well as concerning active ingredients, traditional Chinese patent medicine and Chinese herbs and formulas. Chinese herbal formulas not only stabilize BP but also improve clinical symptoms and quality of life, reverse hypertensionrelated risk factors and protect targeted organs to improve the chances of long-term survival. Thus, therapeutic advantages exist for overall regulation. Importantly, the effectiveness of Chinese herbal formulas with regard to the uncontrollable factors of BP such as insomnia, constipation, mood swings, obesity, pain and so forth will be the focus of future research on Chinese medicine/integrative medicine for treating hypertension. ${ }^{123}$

As previously mentioned, antihypertensive therapy research on Chinese herbal formulas for treating hypertension has made rapid progress over the past 30 years, but certain problems remain that seriously limit the progress of this research; these problems should be solved as soon as possible. Currently, the clinical hypertensive treatment trials using TCM have been limited to small samples of curative effects, and multicenter, large-scale random samples with controlled methods are rarely employed. This limitation leaves the clinical practice of Chinese herbal formulas for treating hypertension short of definitive clinical evidence. Thus, the evaluation criteria of the clinical outcomes of hypertension must also attend to BPV rather than the value of casual BP (clinical BP) as a medical efficacy appraisal standard. Moreover, many experiments have primarily focused on the mechanisms of one aspect of specific Chinese herbal formulas for treating hypertension. The experimental methodology requires rigor, and only a few studies have included in vitro and in vivo samples in the same design.

Because of many of the problems regarding the effectiveness and security of current antihypertensive Western drugs, a great need has arisen to develop efficacious medicines to treat hypertension. Screening highly efficient antihypertensive drugs with fewer adverse effects based on Chinese herbal formulas has attracted much research attention, and the target mechanisms of Chinese herbal formulas for hypertension are a hot topic in the research and development of antihypertensive drugs. Active ingredients with potential antihypertensive effects are the material basis of Chinese herbal formulas for treating hypertension. However, Chinese herbs contain many active ingredients, and a commonly used formula usually contains more than two Chinese herbs. Thus, a large quantity of active ingredients must be identified, extracted and purified from a formula, which is difficult for medical researchers. Furthermore, certain active ingredients are chemically unstable, which limits large-scale synthesis. These pressing issues should be resolved in future research. However, the primary task of the current study was to discuss the antihypertensive effects of Chinese herbal formulas. Multicenter, large-scale random samples using controlled trials are needed to reasonably evaluate the efficacy and safety of Chinese herbal formulas for treating hypertension within the 'concept of holism' and the 'syndrome differentiation' in TCM. ${ }^{124,125}$ Furthermore, the experiments on classic and experienced prescriptions should also be strengthened. By providing intensive research on the antihypertensive effects of Chinese herbal formulas, we can develop many new antihypertensive drugs that possess definite curative effects that target clear mechanisms to significantly advance the research on hypertensive treatment.

\section{CONFLICT OF INTEREST}

The authors declare no conflict of interest.

\section{ACKNOWLEDGEMENTS}

The National Basic Research Program of China (973 Program, No. 2003CB517103) and the National Natural Science Foundation Project of China (No. 90209011) partially supported the current work.

1 Karen S, Simon S, Bernard JG. Hypertension: a global perspective. Circulation 2011; 123: 2892-2896.

2 Chobanian AV, Bakris GL, Black HR, Cushman WC, Green LA, Izzo JL Jr, Jones DW, Materson BJ, Oparil S, Wright JT Jr, Roccella EJ. Seventh report of the Joint National Committee on prevention, detection, evaluation, and treatment of high blood pressure. Hypertension 2003; 42: 1206-1252.

3 Furberg $\mathrm{CD}$, Psaty BM, Pahor M, Alderman MH, Mulrow C. Clinical implications of recent findings from the antihypertensive and lipid-lowering treatment to prevent heart attack trial (ALLHAT) and other studies of hypertension. Ann Intern Med 2001; 135: 1074-1078.

4 Vasan RS, Larson MG, Leip EP, Evans JC, O'Donnell CJ, Kannel WB, Levy D. Impact of high normal blood pressure on the risk of cardiovascular disease. $N$ Engl J Med 2001; 345: 1291-1297.

5 Yikona JI, Wallis EJ, Ramsay LE, Jackson PR. Coronary and cardiovascular risk estimation in uncomplicated mild hypertension: a comparison of risk assessment methods. J Hypertens 2002; 20: 2173-2182.

6 Giovanna L, Francesca V, Roberto P. RAAS inhibition and renal protection. Curr Pharm Des 2012; 18: 971-980.

7 Brown MJ. Aliskiren. Circulation 2008; 118: 773-784.

8 William BW, Bresalier R, Kaplan AP, Palmer BF, Riddell RH, Lesogor A, Chang W, Keefe DL. Safety and tolerability of the direct renin inhibitor aliskiren: a pooled analysis of clinical experience in more than 12,000 patients with hypertension. J Clin Hypertens 2010; 12: 765-775.

9 Musini VM, Fortin PM, Bassett K, Wright JM. Blood pressure lowering efficacy of renin inhibitors for primary hypertension. Cochrane Database Syst Rev 2008; 4; doi:10.1002/14651858.CD007066.pub2.

10 Duprez DA, Munger MA, Botha J, Keefe DL, Charney AN. Aliskiren for geriatric lowering of systolic hypertension: a randomized controlled trial. J Hum Hypertens 2010; 24: 600-608.

11 Sarafidis PA, Bakris GL. Resistant hypertension: an overview of evaluation and treatment. J Am Coll Cardiol 2008; 52: 1749-1757.

12 Elisavet M, Matilda F, Moses SE, Dimitri PM, Evangelos NL. Aliskiren, a direct renin inhibitor, in clinical practice, a new approach in the treatment of hypertension. Curr Vasc Pharmacol 2010; 8: 344-362.

$13 \mathrm{Xu} \mathrm{H}$, Chen KJ. Integrative medicine: the experience from China. J Altern Complement Med 2008; 14: 3-7.

14 Xiong XJ, Chu FY, Li HX, He QY. Clinical application of the TCM classic formulae for treating chronic bronchitis. J Tradit Chin Med 2011; 31: 69-72.

15 Walker AF, Marakis G, Morris AP, Robinson PA. Promising hypotensive effect of hawthorn extract: a randomized double-blind pilot study of mild, essential hypertension. Phytother Res 2002; 16: 48-54.

16 Zhong GW, Chen MJ, Luo YH, Xiang LL, Xie QY, Li YH, Zhang C, Gao F. Effect of Chinese herbal medicine for calming gan and suppressing hyperactive yang on arterial elasticity function and circadian rhythm of blood pressure in patients with essential hypertension. Chin J Integr Med 2011; 17: 414-420.

17 Li H, Liu LT, Zhao WM, Liu JG, Yao MJ, Han YX, Shen YP, Liu XD, Liu L, Wang XM, Cai $\mathrm{LL}$, Guan J. Effects of traditional and integrative regimens on quality of life and early renal impairment in elderly patients with isolated systolic hypertension. Chin J Integr Med 2010; 16: 216-221.

18 Macklin EA, Wayne PM, Kalish LA, Valaskatgis P, Thompsom J, Pian Smith MCM, Zhang Q, Stevens S, Goertz C, Prineas RJ, Buczynski B, Zusman RM. Stop hypertension with the acupuncture research program (SHARP): results of a randomized, controlled clinical trial. Hypertension 2006; 46: 838-845.

19 Li JJ, Lu ZL, Kou WR, Chen Z, Wu YF, Yu XH, Zhao YC and on behalf of the Chinese Coronary Secondary Prevention Study Group. Long-term effects of Xuezhikang on blood pressure in hypertensive patients with previous myocardial infarction: data from the Chinese Coronary Secondary Prevention Study (CCSPS). Clin Exp Hypertens 2010; 32: 491-498. 
20 Stavro PM, Woo M, Heim TF, Leiter LA, Vuksan V. North American Ginseng exerts a neutral effect on blood pressure in individuals with hypertension. Hypertension 2005; 46: 406-411.

21 Li JJ, Lu ZL, Kou WR, Chen Z, Wu YF, Yu XH, Zhao YC and on behalf of the Chinese Coronary Secondary Prevention Study Group. Beneficial impact of Xuezhikang on cardiovascular events and mortality in elderly hypertensive patients with previous myocardial infarction from the China Coronary Secondary Prevention Study (CCSPS). J Clin Pharmacol 2009; 49: 947-956.

22 Liu D, Lin H, Wang H, Zhou W, Chen G, Guo W, Ye J, Xu Z, Chen H, Chen XM, Lai Z, Xiang $\mathrm{C}$. Influence of removing blood stasis and tonifying liver and kidney method on quality of life in patients with essential hypertension: randomized controlled observation. Chin J Clin Rehabil 2006; 10: 9-12.

23 Wang J, Xiong XJ. Control strategy on hypertension in Chinese medicine. Evid Based Complement Altern Med 2012 (doi:10.1155/2012/284847).

24 Wang $H$, Shang HC, Zhang JH, Chen J, Sun BZ, Shang DD, Xiang YZ, Cao HB, Ren $M$, Guo LP, Zhang BL. Niuhuang Jiangya preparation for treatment of essential hypertension: a systematic review. Liaoning Zhong Yi Za Zhi 2008; 35: 649-652.

25 Hu YX. Quantitative Analysis of Clinical Controlled Trials of Traditional Chinese Medicine and Systematic Evaluation of Randomized Controlled Trials Involving Traditional Chinese Medicine for Essential Hypertension. Guangzhou University of Chinese Medicine: Guangzhou, China, 2009.

26 Ren Y, Ou AH, Lin XZ, Lao YR. Meta-analysis of traditional Chinese medicine for essential hypertension. Shanxi Zhong Yi 2006; 27: 794-796.

27 Dong DX, Yao SL, Yu N, Yang B. Systematic review and meta-analysis of Tianma Gouteng Yin combined with enalapril for essential hypertension. Zhongguo Zhong Yi Ji Zheng 2011; 20: 762-764.

28 Zhang HW, Tong J, Zhou G, Jia H, Jiang JY. Tianma Gouteng Yin Formula for treating primary hypertension. Cochrane Database Syst Rev 2012, Art. No.: CD008166; doi:10.1002/14651858.CD008166.pub2, 2012.

29 Xu WJ, Li YL. Systematic review of clinical evidence about calm the liver and subdue yang therapy on the hypertension disease with syndrome of upper hyperactivity of liver yang. Zhonghua Zhong Yi Yao Za Zhi 2012; 27: 736-739.

30 Shi M, Zhang YH. Systematic review of replenishing kidney qi method for essential hypertension with kidney qi deficiency syndrome. Shandong Zhong Yi Za Zhi 2012; 31: 236-238.

31 Wang J, Xiong XJ. Outcome measures of Chinese herbal medicine for hypertension: an overview of systematic reviews. Evid Based Complement Altern Med 2012; 2012: Article ID 697237 (doi:10.1155/2012/697237).

32 Wang J, Yang XC, Feng B, Liu W, Li HX, Li XK, Qian WD, Chu FY, Xiong XJ. Is Yangxue Qingnao Granule combined with antihypertensive drugs, a new integrative medicine therapy, more effective than antihypertensive therapy alone in treating essential hypertension? Evid Based Complement Altern Med 2013; 2013: Article ID 540613 (http://dx.doi.org/10.1155/2013/540613).

33 Xiong XJ, Yang XC, Liu W, Feng B, Ma JZ, Du XL, Wang PQ, Chu FY, Li J, Wang J. Banxia baizhu tianma decoction for essential hypertension: a systematic review of randomized controlled trials. Evid Based Complement Altern Med 2012; 2012: Article ID 271462 (doi:10.1155/2012/271462).

34 Wang J, Yao KW, Yang XC, Liu W, Feng B, Ma JZ, Du XL, Wang PQ, Xiong XJ. Chinese patent medicine liu wei di huang wan combined with antihypertensive drugs, a new integrative medicine therapy, for the treatment of essential hypertension: a systematic review of randomized controlled trials. Evid-Based Complement Altern Med 2012 2012: Article ID 714805 (doi:10.1155/2012/714805).

35 Luiz AB, Cordovil I, Filho JB, Ferreira AS. Zangfu zheng (patterns) are associated with clinical manifestations of zang shang (target-organ damage) in arterial hypertension. Chin Med 2011; 6: 23

36 Georg G, Colombet I, Durieux P, Ménard J, Meneton P. A comparative analysis of four clinical guidelines for hypertension management. J Hum Hypertens 2008; 22: 829-837.

37 Schillaci G, Sacchi N. Diurnal blood pressure variation and left ventricular mass. Am J Cardiol 1996; 77: 325.

38 Qian YS, Zhang WZ, Zhou HF, Wang GL. Relationship of blood pressure variability and TCM constitution classification in essential hypertension patients. Zhongguo Zhong $X_{i}$ Yi Jie He Za Zhi 2003; 23: 88-90.

39 Tao LL, Ma XC, Chen KJ. Clinical study on effect of Qing xuan tiao ya recipe in treating menopausal women with hypertension. Zhongguo Zhong Xi Yi Jie He Za Zhi 2009; 29: 680-684

40 Yu J, Xu FQ. Clinical observation on Qing xuan jiang ya decoction in treatment of essential hypertension with liver-kidney yin-deficiency and liver-yang hyperactivity syndrome. Zhong Xi Yi Jie He Xin Nao Xue Guan Bing Za Zhi 2010; 8: 1-3.

$41 \mathrm{Ji} \mathrm{M}$. Evaluating the efficacy of the bezoar antihypertensive pill by ambulatory blood pressure monitoring. Zhong Cheng Yao 2009; 31: 11-13.

42 Zhao YF, Wu H, Zu HB. Antihypertensive effect of valsartan and levam lodipine besylate combinated with traditional Chinese medicine Song ling xue mai kang therapying patients after stroke evaluated by ambulatory blood pressure monitoring. Shi Yong Xin Nao Fei Xue Guan Bing Za Zhi 2011; 19: 575-577.

43 Fu L, Mao ZX, Wang J, Zheng TR, Wang SL. Effects of Song ling xue mai kang capsule on ambulatory blood pressure in treatment of essential hypertension: a single-blind randomized controlled trial. Zhong Xi Yi Jie He Xue Bao 2009; 7: 509-513.

44 Wang FQ. The effect of Song ling xue mai kang combined with amlodipine on reducing the blood pressure variability in patients with essential hypertension. Jilin Zhong Yi Yao 2011; 31: 149-150
45 Lei ZY, Lin X. Analysis of Banxia baizhu tianma soup on blood pressure variability in menopausal patients with hypertension. Xian Dai Zhong Xi Yi Jie He Za Zhi 2009; 18 449-450.

46 Grassi G, Cattaneo BM, Seravalle G, Lanfranchi A, Mancia G. Baroreflex control of sympathetic nerve activity in essential and secondary hypertension. Hypertension 1998; 31: 68-72.

47 Binggeli C, Corti R, Sudano I, Luscher TF, Noll G. Effects of chronic calcium channe blockade on sympathetic nerve activity in hypertension. Hypertension 2002; 39 892-896.

48 Cai Y, Qi CL, Wu D, Gao L, Lv JH. The protecting effects of berberine on the hypertensive renal impairment in rats fed by enriched high fat-salt-fructose diet. Guangdong Yao Xue Yuan Xue Bao 2011; 27: 65-69.

49 Lu S, Zhang ZB, Gong SY, Su W, Zhu HJ. Effects of isoliensinine on left ventricular hypertrophy and its possible mechanism. Zhong hua Gao Xue Ya Za Zhi 2008; 16: 33-35.

50 Zhou JY, Mo ZX, Zhou SW. Effect of rhynchophy line on central neurotransmitter levels in amphetamine-induced conditioned place preference rat brain. Fitoterapia 2010; 5: 844-848.

51 Yu SY. The effect of Niuhuang jiang ya pills on blood pressure and sympathetic activity. Zhongguo Zhong Yao Za Zhi 2007; 32: 172-175.

52 Fischer JW, Stoll M, Hahnb AW, Ungera T. Differential regulation of thrombospondin1 and fibronectin by angiotensin II receptor subtypes in cultured endothelial cells. Cardiovasc Res 2001; 51: 784-791.

53 Lei Y, Lu QS, Ma XC, Chen KJ. Clinical study on effect of Qing xin capsule in treating patients with hypertension of mild or moderate degree. Zhongguo Zhong Xi Yi Jie He Za Zhi 2005; 25: 114-118.

54 Gildea JJ, Wang X, Jose PA, Felder RA. Differential D1 and D5 receptor regulation and degradation of the angiotensin type 1 receptor. Hypertension 2008; 51: 360-366.

55 Chen Y, Huang YS. Effect of Xia xi oral liquid on blood pressure and the expression of myocardial angiotensin II type 1 receptor in spontaneously hypertensive rats. Zhongguo Lao Nian Xue Za Zhi 2007; 13: 1228-1230.

56 Yu SY. Effects on sympathetic nervous activity of Niuhuang jiang ya pellet on hypertension patients. China J China Mater Med 2004; 29 (Suppl): 116-120.

57 Yu SY, Yu ZA. The effects observation of Niuhuang jiang ya capsule in improving the sex-life quality for hypertension patients. China J China Mater Med 2004; 29 (Suppl): 114-116.

58 Jiang JY, Wang XZ, Luo SS, Wang X, Bian K, Yan Y. Effect of Banxia baizhu tianma decoction on the left ventricular hypertrophy of hypertrophied myocardium in spontaneously hypertensive rat. Zhongguo Zhong Xi Yi Jie He Za Zhi 2010; 30: 1061-1066.

59 Ye XY, Song $\mathrm{H}, \mathrm{Lu} Z \mathrm{ZC}$. Effects of puerarin injection on the mRNA expression of AT1 and ACE2 in spontaneously hypertensive rats. Zhongguo Zhong Xi Yi Jie He Za Zhi 2008; 28: 824-827.

60 Bates DO. Vascular endothelial growth factors and vascular permeability. Cardiovasc Res 2010; 87: 262-271.

61 Tammela T, Enholm B, Alitalo K, Paavonen K. The biology of vascular endothelial growth factors. Cardiovasc Res 2005; 65: 550-563.

62 Herbert SP, Stainier DY. Molecular control of endothelial cell behaviour during blood vessel morphogenesis. Nat Rev Mol Cell Biol 2011; 12: 551-564.

63 Dimmeler S, Zeiher AM. Nitric oxide-an endothelial cell survival factor. Cell Death Differ 1999; 6: 964-968.

64 Abraham D, Dashwood M. Endothelin-role in vascular disease. Rheumatology (Oxford) 2008; 47: 23-24.

65 Zhang ZW, Yue GH, Luo Y. Effects of Huanglian jie du decoction on prothrombotic state in spontaneous hypertension rats. Zhongguo Shi Yan Fang Ji Xue Za Zhi 2011 17: 105-108.

66 Liu JQ, Huang YS. Effect of Xia xi oral liquid on blood pressure, ET, NO and blood viscosity in spontaneously hypertensive rats. Zhongguo Lao Nian Xue Za Zhi 2009; 29: 1816-1817.

67 Chen ZQ, Hong L, Wang H, Yin QL, Lai HL, Lu LX. Effects of Tong xin luo capsule on platelet activating and inflammation factors as well as vascular endothelial function in patients with essential hypertension complicated with diabetes mellitus. Zhongguo Zhong Xi Yi Jie He Za Zhi 2010; 30: 376-379.

68 Yao L, Lv GY. Effect of Zhenge hypertension recipe on nitric oxide and endothelin in spontaneous hypertension rats. Zhongguo Zhong Xi Yi Jie He Za Zhi 2002; 22: 36-37.

69 Zhao YH, Xu YH, Guan Y, Xiang P. Effects of Yi nian jiang ya decoction containing serum on cytokines secretion of vascular endothelium of spontaneously hypertensive rats. Chin J Integr Med 2010; 16: 344-347.

70 Zhao YH, Liu YD, Guan Y, Liu NW. Effects of Yi nian jiang ya decoction on primary hypertension in early stage- a clinical observations on 40 patients. J Tradit Chin Med 2010; 30: 171-175.

71 Song CS, Li H, Wang L, Xu LR, Liu F, Luo ZG. Study on effect and mechanism of aged patients with simple systolic hypertension by Jiangya capsule. Zhongguo Zhong Xi $Y i$ Jie He Za Zhi 2002; 22: 902-905.

72 Li H, Liu F, Cui L, Luo ZG, Liu FZ, Xu LR. Effect of Jiangya capsule on levels of serum nitric oxide synthase activity, malondialdehyde, plasma neuropeptide $Y$ and homocysteine in patients with senile simple systolic hypertension. Zhongguo Zhong Xi Yi Jie He Za Zhi 2003; 23: 898-901.

73 Jin L, Zhou WQ. Clinical study on Jiangya capsule in treating hypertension of aged patients with yin-deficiency and yang excess syndrome. Zhongguo Zhong Xi Yi Jie He Za Zhi 2002; 22: 832-834

74 Jiang W, Zhang WG, Ma XS, Zhou SN. Clinical and experimental study on Jiang zh tiao ya granule in treating essential hypertension and protecting function of vascular endothelium. Zhongguo Zhong Xi Yi Jie He Za Zhi 2002; 22: 18-20. 
75 Cohuet G, Struijker BH. Mechanisms of target organ damage caused by hypertension: therapeutic potential. Pharmacol Ther 2006; 111: 81-98.

76 Tomiyama M, Horio T, Yoshii M, Takiuchi S, Kamide K, Nakamura S, Yoshihara F, Nakahama $\mathrm{H}$, Inenaga K, Kawano Y. Masked hypertension and target organ damage in treated hypertensive patients. Am J Hypertens 2006; 19: 880-886.

77 Odama U, Bakris G. Target organ damage in hypertension. J Clin Hypertens (Greenwich) 2000; 2: 312-318.

78 Leoncini G, Ratto E, Viazzi F, Vaccaro V, Parodi A, Falqui V, Conti N, Tomolillo C, Deferrari G, Pontremoli R. Increased ambulatory arterial stiffness index is associated with target organ damage in primary hypertension. Hypertension 2006; 48: $397-$ 403.

79 Zhang ZG, Wang C, Li L, Fan D, Fu FY, Wu LL. Effects of gadol and ganodcrma spores on the adiponcctin signal pathway in hypcrtrophic myocardium of spontaneous hypcrtcnsive rats. Zhongguo Zhong Xi Yi Jie He Za Zhi 2009; 29: 233-237.

80 Wang S, Wang SR, Zhao YR, Dong AM. Study on effect of Jiang ya tong mai recipe on vascular activating substances in patients of hypertension with left ventricular hypertrophy. Zhongguo Zhong Xi Yi Jie He Za Zhi 2002; 22: 274-276.

81 Wang YH, Xiao WJ, Luo SQ, Wang C, Han JG, Shang T, Wang G. Effects of Jiang ya tong mai formula on blood pressure and left ventricular hypertrophy of spontaneous hypertensive rats. Zhongguo Zhong Xi Yi Jie He Za Zhi 2010; 25: 369-371.

82 Xian SX, Hu SY, Liu XH, Zhao LC, Li NY. Study on effect of Tianma gouteng decoction in intervening myocardial collagen reconstruction in renovascular hypertensive rats. Zhongguo Zhong Xi Yi Jie He Za Zhi 2003; 23: 21-24.

83 Wang SJ, Chen Y, He DD, He LR, Yang YM, Chen JZ, Wang XX. Inhibition of vascular smooth muscle cell proliferation by serum from rats treated orally with Gastrodia and Uncaria decoction, a traditional Chinese formulation. J Ethnopharmacol 2007; 114: 458-462.

84 Ho SC, Ho YF, Lai TH, Liu TH, Su SY, Wu RY. Effect of Tianma Gouteng decoction with subtractive ingredients and its active constituents on memory acquisition. $\mathrm{Am} \mathrm{J}$ Chin Med 2008; 36: 593-602.

85 Yao LM, Liu TW, Wu WF, Zhong GQ. Effects of astragalus injection in reversiong left ventricular hypertrophy induced by renal hypertension in rats. Zhongguo Zhong Xi Yi Jie He Za Zhi 2009; 29: 918-921.

86 Zhou JZ, Lei H, Chen YZ, Li FQ. Effect of erigeron breviscapus injection on ventricular and vascular remodeling in spontaneous hypertension rats. Zhongguo Zhong Xi Yi Jie He Za Zhi 2002; 22: 122-125.

87 Wu DJ, Hong HS, Jiang Q. Effect of Shexiang baoxin pill in alleviating myocardial fibrosis in spontaneous hypertensive rats. Zhongguo Zhong Xi Yi Jie He Za Zhi 2005; 25: 350-353.

88 Chen JY, Fan HL, Zhang SF. Effect of modified Zexie decoction on prevention of kidney injuries of rats with hypertension induced by high-salt diet. Zhong Yi Za Zhi 2012; 53: 234-237.

89 Huang L, Shi ZX, Zhang JL, Mao JS, Luo J. Experimental study on effect of Jiang ya mai jing liquid on spontaneous hypertension rats fed with high lipid diet. Zhongguo Zhong Xi Yi Jie He Za Zhi 2002; 22: 528-530.

90 Chu JF, Wu GW, Zheng GH, Zheng LP, Lin W, Huang MY, Huang YM, Lin RH, Yang ML, Zhou CE. Protective effect of Qing xuan jiang ya decoction on the kidney of spontaneous hypertension rats. Zhong Yi Za Zhi 2012; 53: 420-423.

91 Zhong GW, Li W, Chen MJ, Yi ZJ, Chen GL, Xiang LL. Effects on vascular remodeling and adiponectin expression in aorta in the spontaneously hypertensive rats by Chinese herb mixture method. Zhonghua Gao Xue Ya Za Zhi 2008; 16: 812-816.

92 Wang YX, Dang WY, Chen ZC, Wang SJ, He LR. Comparative study on effects of three therapeutic methods on vascular remodeling in spontaneous hypertension rats. Zhong Yi Za Zhi 2006; 47: 381-383.

93 Jing QW, Chen QL, Deng XG, Cong S, Wu ZZ. Effect of Tong mai ning granule on blood pressure and vascular remodeling in spontaneous hypertensive rats. Zhongguo Zhong Xi Yi Jie He Za Zhi 2002; 22: 34-35.

94 Zhao P, Chen J, Hong YD, Zeng YJ. Ultrasonographic study on effects of Wen dan xie zhuo method in improving vascular endothelial diastolic function and atherosclerosis in hypertension patients with turbid-phlegm syndrome. Zhongguo Zhong Xi Yi Jie He Za Zhi 2007; 27: 21-24.

95 Cheung BM, Li C. Diabetes and hypertension: is there a common metabolic pathway. Curr Atheroscler Rep 2012; 14: 160-166.

96 Whaley CA, Sowers JR. Hypertension and insulin resistance. Hypertension 2009; 54 : 462-464.

97 Cao L, Mao CP, Gu ZL. Effect of puerarin on glucose tolerance and the histomorphology in pancreatic tissue in insulin resistance rats. Zhongguo Xue Ye Liu Bian Xue Za Zhi 2008; 18: 47-49.

98 Zhu QL, He AX, Lv XR. Effects of puerarin on the scavenge of oxygen free radicals and the antagonism against oxidative injury. Jie Fang Jun Yao Xue Xue Bao 2008; 26 : 2478-2480.
99 Wu QF, Wen MX, Lan DH. Effects of Banxia baizhu tianma decoction on salt sensitivity and blood lipid in hypertensive patients with abundant phlegm-dampness syndrome. Fujian Yi Yao Za Zhi 2007; 29: 146-148.

100 Wu QF, Wen MX, Lan DH. Effect of Banxia baizhu tianma decoction on insulin resistance and blood lipid in hypertensive patients with abundant phlegm-dampness syndrome. Fujian Zhong Yi Yao Xue Bao 2007; 17: 8-10.

101 Wang YS, Chen HG, Wu W. Clinical studies of Bu shen yi xin tablets on improving the insulin resistance in patients with essential hypertension. Xin Zhong Yi 2003; 35: 42-43.

102 Kwan CY, Achike F. Tetrandrine and related bis-benzylisoquinoline alkaloids from medicinal herbs: cardiovascular effects and mechanisms of action. Acta Pharmacol $\operatorname{Sin} 2002$; 23: 1057-1068.

103 Lv GY, Chen SH, Lv HJ, Chen Y. Henostatic studies on anti-hypertensive effect of Xueling. Zhejiang Zhong Yi Xue Yuan Xue Bao 2003; 27: 47-49.

104 Weng JL. Song ling xue mai kang on hemorheology in patients with hypertension. Shi Yong Xin Nao Xue Guan Za Zhi 2006; 14: 885-886.

105 Ren JX, Lin CR, Wang M, Xu H, Liu JX. The effect and mechanism of Niuhuang jiang ya capsule on the function of platelets in rats. Zhongguo Shi Yan Fang Ji Xue Za Zhi 2007; 13: 34-36

106 Liu JQ, Li HZ, Cui ZW, Huang SZ. Effect of Song ling xue mai kang on high-sensitivity $C$ reactive protein and carotid artery atherosclerosis in hypertensive patients. Zhong $X i$ Yi Jie He Xin Nao Xue GuanBing Za Zhi 2011; 9: 266-267.

107 Kang YF, Zhang AM, Shu C. Effect of Niuhuang jiang ya pill on atherosclerosis. Jilin Zhong Yi Yao 2009; 29: 1044-1045.

108 Chen KJ. Clinical service of Chinese medicine. Chin J Integr Med 2008; 14: 163-164.

109 Chen KJ. Where are we going? Chin J Integr Med 2010; 16: 100-101.

110 Robinson N. Integrative medicine-traditional Chinese medicine, a model? Chin J Integr Med 2011; 17: 21-25.

111 Xiong XJ, Yang XC, Feng B, Liu W, Duan L, Gao A, Li HX, Ma JZ, Du XL, Li N, Wang $P Q$, Su KL, Chu FY, Zhang GH, Li XK, Wang J. Zhen gan xi feng decoction, a traditional Chinese herbal formula, for the treatment of essential hypertension: a systematic review of randomized controlled trials. Evid Based Complement Altern Med 2013; 2013: Article ID 982380 (http://dx.doi.org/10.1155/2013/982380).

$112 \mathrm{Xu} \mathrm{H}$, Chen KJ. Complementary and alternative medicine: is it possible to be mainstream? Chin J Integr Med 2012; 18: 403-404.

$113 \mathrm{Xu} \mathrm{H}$, Chen KJ. Making evidence-based decisions in the clinical pratice of integrative medicine. Chin J Integr Med 2010; 16: 483-485.

114 Liu MY, Chen KJ. Convergence: the tradition and the modern. Chin J Integr Med 2012; 18: 164-165.

115 Lee MS, Pittler MH, Taylor-Piliae RE, Ernst E. Tai chi for cardiovascular disease and its risk factors: a systematic review. J Hypertens 2007; 25: 1974-1975.

116 Lee MS, Choi TY, Shin BC, Kim JI, Nam SS. Cupping for hypertension: a systematic review. Clin Exp Hypertens 2010; 32: 423-425.

117 Lee MS, Lee MS, Choi ES, Chung HT. Effects of qigong on blood pressure, blood pressure determinants and ventilatory function in middle-aged patients with essential hypertension. Am J Chin Med 2003; 31: 489-497.

118 Lee MS, Lee EN, Kim JI, Ernst E. Tai chi for lowing resting blood pressure in the elderly: a systematic review. J Eval Clin Pract 2010; 16: 818-824.

$119 \mathrm{Kim}$ JI, Choi JY, Lee H, Lee MS, Ernst E. Moxibustion for hypertension: a systematic review. BMC Cardiovasc Disord 2010; 10: 33

120 Lee MS, Lim HJ, Lee MS. Impact of qigong exercise on self-efficacy and other cognitive perceptual variables in patients with essential hypertension. J Altern Complement Med 2004; 10: 675-680.

121 Lee MS, Pittler M, Guo RL, Ernst E. Qigong for hypertension: a systematic review of randomized clinical trials. J Hypertens 2007; 25: 1525-1532.

122 Wang J, Wang PQ, Xiong XJ. Current situation and re-understanding of syndrome and formula syndrome in Chinese medicine. Intern Med 2012; doi:10.4172/21658048.1000113.

123 Wang J, Xiong XJ. Current situation and perspectives of clinical study in integrative medicine in China. Evid Based Complement Altern Med 2012; 2012: Article ID 268542 (doi:10.1155/2012/268542).

$124 \mathrm{Xu} \mathrm{H}$, Chen KJ. Integrating traditional medicine with biomedicine towards a patientcentered healthcare system. Chin J Integr Med 2011; 17: 83-84.

125 Jiang WY. Therapeutic wisdom in traditional Chinese medicine: a perspective from modern science. Trends Pharmacol Sci 2005; 26: 558-563.

cc) (1) $(9)$ This work is licensed under a Creative Commons cc. Attribution-NonCommercial-NoDerivs 3.0 Unported License. To view a copy of this license, visit http://creativecommons. org/licenses/by-nc-nd/3.0/ 